\title{
Comparative Gene Analysis in Chordate Embryonic Development Reveals Co-existence of Von Baer's and Haeckel's Postulates
}

\section{Song Guo}

Skolkovo Institute of Science and Technology

Haiyang Hu

CAS Key Laboratory of Computational Biology, CAS-MPG Partner Institute for Computational Biology

Chuan Xu

CAS Key Laboratory of Computational Biology, CAS-MPG Partner Institute for Computational Biology

Naoki Irie

University of Tokyo https://orcid.org/0000-0002-6720-381X

Philipp Khaitovich ( $\nabla$ khaitovich@eva.mpg.de)

Skolkovo Institute of Science and Technology

Article

Keywords: evolution, evo-devo, Haeckel, von Baer, development

Posted Date: June 17th, 2021

DOI: https://doi.org/10.21203/rs.3.rs-579345/v1

License: (c) (i) This work is licensed under a Creative Commons Attribution 4.0 International License.

Read Full License 
1 Comparative gene analysis in chordate embryonic development

2 reveals co-existence of Von Baer's and Haeckel's postulates

3

4 Song Guo ${ }^{1,2, \dagger}$, Haiyang $\mathrm{Hu}^{2, \dagger}$, Chuan $\mathrm{Xu}^{2, \dagger}$, Naoki Irie ${ }^{3,4}$, Philipp Khaitovich ${ }^{1, *}$ 5

$6 \quad \quad{ }^{1}$ Skolkovo Institute of Science and Technology, 121205, Moscow, Russia

$7 \quad{ }^{2}$ CAS Key Laboratory of Computational Biology, CAS-MPG Partner Institute for

8 Computational Biology, Shanghai Institute for Nutrition and Health, CAS, 320 Yue

$9 \quad$ Yang Road, 200031, Shanghai, China.

$10 \quad{ }^{3}$ Department of Biological Sciences, University of Tokyo, Tokyo, Japan.

$11{ }^{4}$ Universal Biology Institute, The University of Tokyo, Tokyo, Japan.

$13 \dagger$ Equally contributed to this work.

$14{ }^{*}$ Correspondence should be addressed to: P.Khaitovich@skoltech.ru 


\section{Abstract}

22 The relationship between embryonic development and evolution historically

23 investigated based on embryo morphology could now be reassessed using mRNA

24 expression endophenotype. Here, we analyzed the conservation of divergence of the

25 developmental mRNA expression profiles in nine evolutionarily distinct species, from 26 oyster to mouse, and compared them to the original concepts formulated by von Baer 27 and Haeckel. We find nearly linear conservation of species' developmental programs 28 among these species, compatible with models rooted on von Baer's postulates, for

29 approximately a third of expressed orthologous genes. By contrast, $5-15 \%$ of 30 developmental expression profiles, enriched in neural genes, displayed an alignment

31 pattern compatible with the terminal edition paradigm proposed by Haeckel. Thus, the 32 development-evolution relationship based on mRNA expression agrees with early 33 concepts based on embryo morphology and demonstrates that the corresponding 34 patterns coexist in chordate development.

36 Keywords: evolution, evo-devo, Haeckel, von Baer, development 


\section{Introduction}

39 The general relationship between ontogeny and phylogeny, or development and 40 evolution, has long been discussed. Among cornerstones of the evolutionary 41 developmental theory are the arguments formulated in von Baer's laws of embryology 42 and Ernst Haeckel's Biogenetic law. Although von Baer did not accept the concept of 43 evolution, his idea that the earlier stages of embryogenesis reflect shared traits of a 44 broader taxonomic group ${ }^{1}$ laid the foundation for the current evolutionary views on 45 developmental programs' conservation. Ernst Haeckel, who advocated evolution, 46 proposed in his Biogenetic law that the embryogenesis replays the species' 47 evolutionary past. Thus, according to this concept, the new developmental stages will 48 be added to the ancestral embryonic program to produce more recently evolved 49 species $^{2}$. After a long debate, the general concept of early embryogenesis 50 conservation, reflecting a more ancestral stage, continued in a form of a monotonic

51 developmental conservation model known as the funnel model. More recently, a 52 developmental conservation model called the developmental hourglass model was 53 proposed, which placed the most significant stage conservation at a mid-embryonic 54 part of embryogenesis, defined as the phylotypic period ${ }^{3}$. The applicability of the 55 hourglass model to the conservation of developmental stages at mRNA expression 56 level has been supported both in animals s, 5, 6, 7,8 and plants $^{9,10,11}$. Recent studies, 57 however, demonstrated the applicability of the funnel model or the coexistence and 58 validity of both funnel and hourglass models at different levels of trait evolution in 


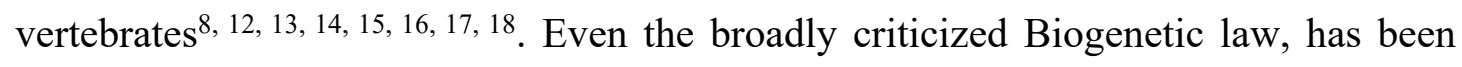
pointed out to have potential applicability of its principles ${ }^{19}$.

One of the major barriers in testing the relationship between development and evolution, including the applicability of concepts proposed in Biogenetic law or the von Baer's law of embryology, is the difficulty in identifying evolutionarily-homologous developmental stages among distant species. While

65 pioneering studies focused on the investigation of embryonic morphology ${ }^{20}$, recent 66 works relied on developmental changes in mRNA expression profiles as an 67 informative endophenotype ${ }^{16}, 21,22,23$. The use of gene expression facilitates inter-species comparisons, as orthologous genes can be matched among species, and their expression profiles could be traced at all stages of development. Several studies examined developmental gene expression patterns in mammals $^{24}$, vertebrates ${ }^{16}$,

71 chordates $^{17,22}$, and fruit fly species ${ }^{25}$. These studies, however, either focused on gene 72 expression patterns within a specific species or compared developmental stage 73 conservation within the concepts of the hourglass model paradigm.

Here, we compared developmental expression patterns among eight chordate species and oyster to directly investigate the compatibility of developmental expression patterns with predictions of the Biogenetic law and von Baer's law of 77 embryology. To enable such a comparison, we formulated a "continuous" developmental model describing the ontogenetic gene expression pattern alignment,

79 which presumes the existence of a general developmental program spanning the entire 
development, as suggested by the Baer's law and its later modifications. By contrast, an alternative "progressive" developmental model describes the developmental gene expression profiles of a species with more ancestral body plan organization align the

83 best to early embryonic stages of species with more complex structural organization, as 84 mentioned in Haeckel's Biogenetic law.

\section{Results}

\section{Alignment of developmental gene expression patterns among species}

We investigated the relationship between ontogeny and phylogeny of chordate species at the level of molecular phenotype using RNA-sequencing (RNA-seq) data collected over the entire course of embryonic development in eight chordate species of different organizational complexity (amphioxus, ciona, zebrafish, two species of frogs, turtle, chicken, and mouse) and an outgroup species (oyster) ${ }^{8,26}$. In each species, the data were collected at 11 to 20 developmental stages and measured in duplicates (Fig. 1a; Supplementary Data 1 and Supplementary Fig. 1).

Within species, differences among developmental stages explained approximately 90\% of total expression variation (Fig. 1b,c and Supplementary Fig. 2). By contrast,

97 other factors, such as biological replicates and sequence coverage, explained $1 \%$ and $98 \quad 0.6 \%$ of variation each (Fig. 1b). Accordingly, on average, $85 \%$ of detected genes 99 showed significant expression differences among developmental stages in each 

corrected $p<0.05$; Fig. 1d and Supplementary Data 2).

We searched for the best temporal alignment between each pair of species using genes preferentially expressed at a particular developmental stage (stage-associated genes; Methods; Supplementary Data 3) ${ }^{21}$. Surprisingly, despite substantial differences in organizational complexity, an approximately linear alignment of

106 developmental stages was a dominant stage-matching pattern in each pair of species

107 (Fig. 1e and Supplementary Fig. 3,4).

\section{Visualization of developmental expression patterns}

109 We next investigated developmental expression trajectories of the 244 orthologous

110 genes classified as development-related in all nine species using the nearly linear,

111 species' alignment (Fig. 2a). These genes were grouped into six clusters based on

112 their developmental profiles in the unsupervised clustering analysis (Fig. 2b).

113 Remarkably, $66 \%$ of the genes fell within a single cluster representing a decreasing

114 expression pattern conserved across all nine species (CL9.1) (Fig. 2c). By contrast,

115 the expression trajectories in the other clusters differed more among species, with the

116 extent of differences being directly proportional to corresponding phylogenetic 117 distances (Fig. 2e and Supplementary Fig. 5).

118 The same analysis conducted using 2,038 development-related orthologs shared

119 among six vertebrate species revealed a CL9.1-like developmental pattern represented 120 by a single cluster (CL6.1) (Fig. 2d). Genes in clusters CL9.1 and CL6.1 overlapped 
121 significantly and were enriched in similar biological processes, including spliceosome

122 and RNA processing (Fig. 2f and Supplementary Data 4).

123 The second-largest cluster found in vertebrates (CL6.2) showed an opposite,

124 ascending expression pattern conserved among species and contained genes enriched

125 in signaling pathways, such as cGMP-PKG signaling pathway, oxytocin signaling

126 pathway, and Renin secretion (Supplementary Data 4). Notably, this cluster

127 overlapped with the chordate cluster CL9.4, where the ascending pattern was

128 conserved among the seven species, excluding ciona and oyster (Fig. 2c). By contrast,

129 genes in other clusters showed developmental profiles divergent among species,

130 including patterns involving partially similarity, development shifts, and inversions.

\section{Two alternative evolutionary models of species’ development}

132 We next sought to test, from the perspective of gene expression, two models, the

133 continuous and progressive, describing embryogenesis rooted in von Baer's and

134 Haeckel's ideas. Specifically, developmental concepts derived from von Baer's ideas,

135 such as the hourglass and the funnel models, presume general preservation of the

136 developmental profiles across species (Fig. 3a). Our continuous model (CM) follows

137 this assumption, predicting the best alignment between complete developmental

138 expression profiles of the two species. Alternatively, based on the Haeckel's postulate

139 that embryogenesis replays the evolutionary history of the species, we proposed a

140 progressive model (PM), on which the developmental gene expression profiles of a

141 species with more ancestral body plan organization will align the best to early 
142 embryonic stages of species with more recently evolved organization, "replaying" the 143 evolutionary history of this body plan (Fig. 3a; Supplementary Fig. 6).

144 We found that out of all development-related genes that aligned significantly to 145 the mouse developmental trajectory, $19-36 \%$ aligned linearly, thus behaving in 146 agreement with the continuous model predictions (CM genes) (correlation test, $r>0.7$, $147 p<0.05$; Fig. 3b,e; Supplementary Fig. 7 and Supplementary Data 5). By contrast, $1485-15 \%$ of development-related genes aligned significantly better to the truncated sets 149 (PM genes) (permutations, BH-corrected $p<0.05$; Fig. 3b,c,e; Supplementary Fig. 7 150 and Supplementary Data 6). Further, in each pairwise alignment, PM genes did not 151 align uniformly to all shortened mouse developmental intervals but tended to peak at 152 a particular fragment. For instance, oyster PM genes aligned best to the mouse 153 developmental fragments ending at stages 3-4, while amphioxus PM genes - to the 154 fragments ending at stages 5-6 (Fig. 3b,c and Supplementary Fig. 8). Overall, PM 155 genes in species evolutionarily proximal to the mouse tended to align to increasingly 156 longer truncated mouse developmental sets (Fig. 3d). This phylogenetic ordering of 157 ontogenetic patterns was not caused by the alignment procedure, which was not 158 biased to any developmental stage, and robust to the use of evolutionarily old genes 159 present in each of the nine investigated species (Supplementary Fig. 9). By contrast, 160 repeating the alignment procedure using species with more ancient body plans, such 161 as amphioxus or ciona, instead of the mouse, did not yield a consistent significant 162 excess of genes following PM predictions (Supplementary Fig. 10). This phenomenon 
suggested that genes conforming to PM predictions demonstrate the

164 phylogeny-ontogeny relationship aligning with the original Haeckel's ideas, even

165 though we did not include this aspect of the Biogenetic law in PM model formulation.

\section{Properties of CM and PM genes}

167 While we defined CM and PM gene sets independently for each species, each set

168 overlapped significantly between species (Methods, subsampling,

169 Bonferroni-corrected $p<0.05$; Supplementary Data 7), indicating the conservation of

$170 \mathrm{CM}$ and PM patterning across chordate embryogenesis and presumably convergent

171 functionality specific to each model.

172 Indeed, CM genes showed enrichment in Gene Ontology (GO) terms corresponding

173 to general cellular functions, such as spliceosome, RNA transport, DNA replication,

174 and several metabolic processes (Hypergeometric test, $p<0.05$; Supplementary Table

175 8). By contrast, PM genes were primarily enriched in neural functions, including axon

176 guidance, glutamatergic synapse, dopaminergic synapse, and MAPK signaling

177 pathways (Hypergeometric test, $p<0.05$; Fig. 3f and Supplementary Data 8). In line

178 with functional enrichment results, expression of $\mathrm{CM}$ genes displayed significantly

179 lower tissue-specificity in mouse ${ }^{27}$ compared to PM genes, and all expressed genes

180 (one-sided Fisher's exact test, $p<0.05$; Supplementary Fig. 11). Nonetheless, PM

181 genes were more conserved at the amino acid sequence level compared to CM genes

$182(\mathrm{Ka} / \mathrm{Ks}$, one-sided Wilcoxon rank-sum test, $p<0.0005)$ (Fig. 3g and Supplementary

183 Fig. 12,13). Accordingly, CM genes substantially overlapped with CL9.1 genes 
184 (one-sided Fisher's exact test, odds ratio $=3, p<0.0001$ ) and were involved in the

185 same biological processes as CM genes, including spliceosome and RNA processing

186 (Supplementary Data 9).

\section{Discussion}

188 Our goal was to explore the general relationship between ontogeny and phylogeny

189 based on the comparison of developmental gene expression trajectories among nine

190 species separated by approximately 800 million years of evolution. Our results

191 indicate that the continuous and progressive developmental patterns rooted in von

192 Baer's and Haeckel's ideas match approximately half of all detected mRNA

193 developmental expression profiles. Between the two models, the continuous

194 developmental model consistent with Baer's arguments was nearly three-fold more

195 prominent compared to the progressive model compatible with the Biogenetic law.

196 These results do not conflict with the hourglass model of development. Our previous

197 work demonstrated the applicability of this model to all nine species' development ${ }^{8}$.

198 While the hourglass model compares relative conservation of developmental stages,

199 we focused on the alignment of expression differences among stages.

200 Expression profiles conserved along the entire species' development included

201 two main patterns: a gradual decrease conserved among all nine species and gradual

202 increase conserved among six vertebrates. The conserved downward pattern coincides

203 with the reported preferential expression of evolutionary older genes at earlier

204 developmental stages ${ }^{28}$. Consistently, genes forming this pattern tend to display 
ubiquitous expression among tissues and are involved in essential functions, such as

206 RNA processing. Further, a study examining embryonic gene expression reported the existence of such a pattern in isolated mouse tissues ${ }^{29}$, indicating that the descending

208 expression reflects alterations within embryo tissues rather than changes in embryo

209 composition. Overall, expression patterns conserved between species over the entire

210 development involve $19-36 \%$ of orthologous development-related genes detected in

211 our study, constituting the major alignment trend.

212 The second prominent type of developmental relationship among species,

213 involving 5-15\% of orthologous development-related genes, reflected the predictions

214 of Haeckel's Biogenetic law. Consistent to Haeckel's views, the best developmental

215 alignment matched the stages reflecting the phylogenetic history of the species, rather

216 than the entire developmental span. When aligning developmental profiles of eight

217 species to the mouse, we indeed observed this phenomenon. First, for this gene group,

218 the developmental expression profiles indeed aligned the best to the truncated mouse

219 developmental series lacking late stages. Second, for each species, these alignments

220 peaked at a particular shortened mouse developmental fragment, with species

221 phylogenetically more distant from the mouse aligning best to the increasingly shorter

222 mouse developmental sets. Intriguingly, while all species evolved their developmental

223 programs after branching from the common ancestor, a mirror procedure, alignment

224 to the fragmented developmental series of amphioxus and ciona representing more

225 ancient body plans, revealed potential PM genes in only two of 16 alignments 
226 (Supplementary Fig. 10). Further, there was no relationship between the length of the 227 best-aligning developmental fragment and the phylogenetic distance between species.

228 Similar to the linearly-aligning genes, the expression behavior of progressive 229 genes overlapped significantly between species (Supplementary Data 7), indicating

230 their potential functional conservation. These genes were indeed more conserved at 231 the amino acid sequence level than the linearly-aligning genes and displayed 232 enrichment in specialized functions, such as synaptic signaling and secretion.

233 Our work highlights the usefulness of quantitative endophenotypes as an essential 234 complement to morphology-based developmental studies. It further suggests partial 235 applicability of Haeckel's ideas to a fraction of developmental expression differences 236 involving genes associated with neural functions.

\section{Methods}

$239 \quad$ RNA-seq data processing

240 All data in this analysis were downloaded from $\mathrm{Hu}$ et al. ${ }^{8}$. We adopted the same 241 methods to quantify gene expression. In brief, we got the RNA-seq data of 242 Crassostrea gigas from GEO database (accession number SRP014559) ${ }^{26}$, and the rest 243 of species from DDBJ (accession number DRA003460) (Supplementary Data 1). To 244 quantify gene expression, we mapped RNA-seq reads to the corresponding genome 245 (Supplementary Data 1) using Tophat (v2), allowing up to three mismatches and 246 indels, except for ciona (Ci). In the case of ciona, we mapped reads to the genome 
247 with up to five mismatches and indels as the ciona RNA-seq data and the genome data 248 have slightly lower quality compared to the rest.

249 To define the expressed genes, we required the maximal expression across all 250 development stages exceeding 1 FPKM. For each stage, we calculated expression as

251 the mean of expression of the replicated samples. More than $70 \%$ of coding genes 252 annotated for a given species were reliably detected, except for amphioxus (41\%) 253 (Supplementary Data 10).

\section{Identification of development-related genes}

255 We defined developmental alterations of gene expression levels using polynomial 256 regression models following the method described in Somel et al. ${ }^{30}$. For each gene, we 257 chose the best regression model with the developmental stage (by rank) as predictor 258 and expression level as a response with Benjamini-Hochberg corrected $p<0.05$. The 259 genes that fit a significant regression model were termed as development-related genes 260 (Supplementary Data 2).

\section{Identification of stage-associated genes}

262 We defined genes preferentially expressed at a particular developmental stage as 263 stage-associated genes in each species following the method described in ${ }^{8,21}$. For each 264 species, we required FPKM (fragments per kilobase of exon model per million reads 265 mapped) $>2$ at a particular stage and Z-score (normalized FPKM across samples) 266 representing the difference between this stage and the rest of stages $>1.5$. On average, 
$65 \%-90 \%$ of genes expressed in development were identified as stage-associated genes (Supplementary Data 3).

Alignment of mouse developmental stage sets to developmental profiles of other species

270 To assess the transcriptome similarity in the course of developmental stages between

271 mouse $(\mathrm{Mm})$ and other species, we used stage-associated and 1:1 orthologous gene to

272 align the developmental stages between mouse and other species followed by the 273 method reported in $^{8,21}$. The detailed description can be found in the supplementary 274 materials of ${ }^{8,21}$. In brief, we calculated the pairing score using the hypergeometric test 275 to evaluate the ratio of orthologs overlapping within the stage-associated genes 276 pairwise. The pairing score can be written as:

$$
\text { Paring score }=-\log _{10}(\text { Bonferroni corrected P-values })
$$

278 The paring score was used to quantify the significance of the overlap on each pairwise 279 stage comparison between the mouse and another species. From the paring score, we 280 identified the relationship between mouse and other species (Fig. 1e). To check the 281 stability of this relationship, we repeated the comparison 500 times with randomly 282 assigned stage-associated genes for each stage of each species using the same 283 procedure. 


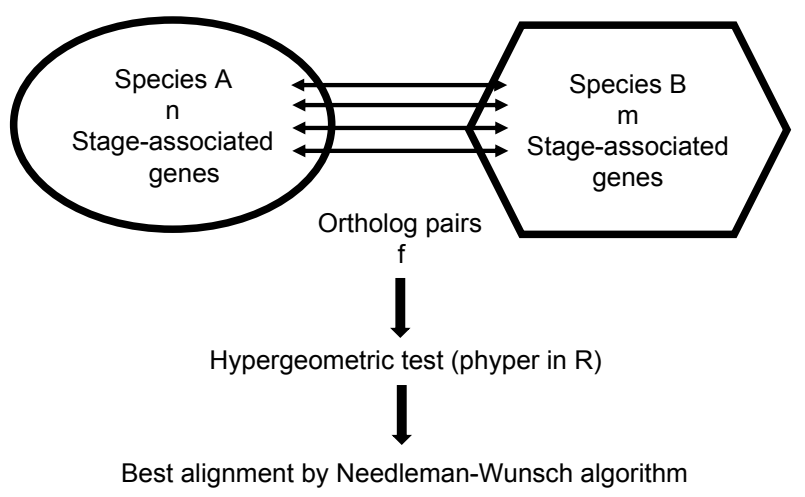

285 According to the above scheme, we further assigned the corresponding stage 286 alignment between mouse and other species using the Needleman-Wunsch algorithm 287 with the gap penalty equal to one. To estimate the stability of alignment based on the 288 mean of the replicates, we randomly chose one individual per stage, aligned two 289 species 500 times, and calculated the frequency for each pair of alignment. The 290 resulting frequency was presented by the thickness of the line in Fig. 2a.

292 To identify genes with expression trajectories compatible with the continuous model $293(\mathrm{CM})$ or progressive model (PM) predictions, we considered development-related 294 genes as mentioned in "Identification of development-related genes" with existing 295 orthologs between mouse and any other species. We then linearly aligned the 296 complete set of developmental stages in a given species (oyster, amphioxus, ciona, 297 zebrafish, frogs, turtle, and chicken) to the increasingly truncated sets of mouse 298 developmental stages, i.e., from first two to all 17 stages (Supplementary Fig. 6). To 299 this end, we interpolated 20 time points uniformly distributed across the whole 300 ontogeny of non-mouse species using cubic smoothing spline (with three degrees of 
301 freedom for amphioxus data and six degrees of freedom for other species) or 302 polynomial regression curves (up to the fourth degree). The two methods for 303 interpolating expression values across development were used to ensure the 304 robustness of our CM or PM predictions. Of note, during cubic smoothing spline, the 305 degrees of freedom chosen for amphioxus were fewer than other species, due to the 306 relatively fewer sampled developmental stages of this species; whereas during 307 polynomial regression, we chose up to four degrees to take advantage of the 308 near-fitness of polynomial regression in most time series data.

309 We then aligned the resulting expression trajectories to the gradually increasing

310 sets of mouse developmental stages, where the expression trajectory was interpolated 311 using the same approach (cubic smoothing spline with six degrees of freedom or 312 polynomial regression with up to the fourth degree). The alignments with the Pearson 313 correlation coefficient (PCC) greater than 0.7 and correlation test $p$-value less than 3140.05 were considered as valid. We further selected the alignment with the maximum

315 PCC among all valid alignments as the ultimate one. Genes failing to pass the two 316 alignment criteria were considered as non-aligned, regardless of the complete or 317 partial linear alignment paradigms.

318 Based on the above classification procedure, we summarized for each species, the 319 number of genes aligning to each set of developmental stages of the mouse. 320 Furthermore, we repeated the classification process by shuffling the orthologous 321 relationships between non-mouse species and the mouse (Supplementary Fig. 7). 
322 Specifically, we expected that by shuffling, each developmental stage of the mouse 323 would be equally represented in other species, allowing us to calculate the random 324 expectation of alignment occurrences for each stage. Thus, the final number of genes 325 aligning to each set of developmental stages of the mouse was obtained after 326 subtracting these background contaminations. Genes adhering to the continuous 327 model (CM) were defined as those best aligned between mouse and other species over 328 the complete developmental intervals. By contrast, genes following the progressive 329 model (PM) were defined by the following criteria: i) aligned best to a truncated set of

330 mouse developmental stages; ii) displayed a significantly greater number of aligning 331 genes compared to the background distributions, determined using a $\mathrm{BH}$-corrected $332 p$-value of less than 0.05 as a cutoff; and iii) an increase in the number of genes 333 aligning to particular sets of mouse stages compared to its adjacent sets (Fig. 3a, 334 Supplementary Data 5 and Supplementary Data 6).

To further check the robustness of the alignment results, we restricted our 336 analysis to evolutionarily old genes. To do so, we repeated the alignment procedure 337 using a subset of the development-related genes with inferred Earliest Ortholog Level 338 (EOL) < level 10. This conservation level corresponds to genes that appeared and 339 became fixed within the genomes before the separation of Chordata ${ }^{31}$.

340 Overlap of CM/PM genes between species

341 To calculate the significance of the overlap of CM or PM genes between species, we 342 sampled the same number of $\mathrm{CM}$ or $\mathrm{PM}$ genes in each species from all 
343 development-related genes 1,000 times and recalculated the number of overlapping

344 genes to obtain the empirical distribution. The overlap significance $p$-value was

345 calculated as the proportion of the values, which were as larger or greater than the

346 actual overlapping gene number. Given all pairs of species involved, a

347 Bonferroni-corrected $p$-value of less than 0.05 was used as the cutoff of the overlap

348 significance (Supplementary Data 7).

349 Amino acid conservation of CM/PM gene

350 We compared the evolutionary conservation between proteins encoded by CM and $351 \mathrm{PM}$ genes using the $\mathrm{Ka} / \mathrm{Ks}$ metric $^{32}$. Since in this study, we are not studying $\mathrm{Ka} / \mathrm{Ks}$ 352 variations across the evolutionary branches - we focused on the comparisons of PM 353 versus $\mathrm{CM}$ genes within each individual species, we conducted this analysis mainly in 354 a pairwise manner between a given species and mouse. Firstly, we extracted the 355 coding sequences of each gene based on the corresponding annotation information 356 and then translated the sequences into amino acid sequences using the function 357 "translate" implemented in the Bioconductor package "Biotrings". The longest protein 358 sequence was selected as the gene protein sequence. Next, we performed protein 359 sequence alignments between non-mouse species and the mouse using the function 360 "pairwiseAlignment" in "Biostrings", with the scoring matrix set as BLOSUM50. The 361 resulting protein alignment was translated to the nucleotide level and used as the input 362 for $\mathrm{PAML}^{33}$ for a $\mathrm{Ka} / \mathrm{Ks}$ analysis output. This procedure was conducted in a pairwise 363 manner for the mouse and a given non-mouse species to match the corresponding 
364 definitions of PM and CM genes carried out in a pairwise manner (Supplementary Fig.

365 12). To test the robustness of our $\mathrm{Ka} / \mathrm{Ks}$ calculations in the context of multiple species, 366 we performed multiple sequence alignment using Clustal Omega ${ }^{34}$ for all protein 367 sequences mentioned above convert to nucleotide levels. We next utilized the

368 CODEML application in PAML to calculate the $\mathrm{Ka} / \mathrm{Ks}$ for given cross-species 369 ortholog. The significance of the difference between PM and CM genes in each 370 species was assessed using one-sided Wilcoxon rank-sum test (Supplementary Fig. 371 13).

372 Gene expression interpretation to mouse developmental stage

373 To compare the similarity of the expression profiles across developmental stages of

374 nine species, we used the predicted developmental stage alignment presented in Fig. 2a 375 to create a unified alignment of eight species to the mouse developmental curves. To do 376 so, we mapped 33 stages cumulatively interpolated from eight species to the full mouse 377 developmental curve fitted using cubic smoothing spline with ten degrees of freedom.

378 We then compared gene expression curves among nine species based on z-transformed 379 expression of each gene interpolated at these 33 stage points.

380 Clustering of gene expression profiles in six vertebrate and nine chordate species

381 To investigate the expression pattern diversity in nine or six species, we used 382 hierarchical clustering (hclust function in $\mathrm{R}$ ) of $\mathrm{z}$-transformed gene expression 383 trajectories aligned among species with (1 - rho) as the distance measure, where rho is 
384 the Spearman correlation coefficient. We chose k equal six, as optimal, based on visual 385 inspection of clusters obtained using different $\mathrm{k}$ values.

Functional annotation of developmental expression patterns

387 To check the functions of genes in each cluster, we applied Gene Ontology (GO) and

388 Kyoto Encyclopedia of Genes and Genomes (KEGG) $)^{35}$ enrichment tests. GO 389 annotation of mouse genes was downloaded from Bioconductor package 390 "org.Mm.eg.db". Mouse pathway annotation was downloaded from 391 http://rest.kegg.jp/list/pathway/ and http://rest.kegg.jp/link/genes/.

For GO enrichment test, the "elim" algorithm of topGO ${ }^{36}$ was chosen to eliminate

393 the hierarchical dependency of the GO terms. Fisher's exact test was applied for each 394 GO term. The background set consisted of all development-related genes orthologous 395 among six vertebrate species (2,038 genes) and nine chordate species (244 genes), 396 respectively. For the test of vertebrate gene clusters, Benjamini-Hochberg correction 397 on "molecular function", "biological process", and "cellular component" was applied 398 independently. GO terms with $\mathrm{BH}$ corrected $p<0.05$ were reported. We applied no 399 multiple test correction to chordate' gene cluster enrichment analysis due to low 400 statistical power of the dataset and used a more stringent nominal $p$-value cutoff of $p$ $401<0.001$

402 For the pathway enrichment test, the reference genes were the same as for 403 GO-based analysis. We used hypergeometric test (phyper in R) to assess the 404 enrichment in each KEGG pathway. Bonferroni correction was applied for genes in 
vertebrate clusters. Pathways with corrected $p<0.05$ were reported as significantly

406 enriched. No correction was applied to genes from chordate clusters due to low

407 statistical power, and the nominal pathway enrichment $p$-value was set to $p<0.05$. This

408 relaxed cutoff was used to assess the potential overlap between enriched functions

409 found in vertebrate and chordate clusters (Supplementary Data 4).

\section{Species tree construction}

411 The species tree was generated under the web https://phylot.biobyte.de/ with NCBI

412 taxonomy IDs (10090, 9031, 13735, 8364, 7955, 7719, 8355, 7739, 29159).

413 The species separation time to the mouse was obtained from

414 http://www.timetree.org $/ 37$.

416 Analyses were conducted in the $\mathrm{R}$ environment (http://www.r-project.org/). To

417 minimize the type I error rate, we used multiple test correction for $p$-value calculations,

418 unless specifically indicated otherwise. The statistically significant level used for each

419 was specified in the main text. Additionally, we used Perl, Python, and R packages, 420 including "topGO", "reshape", "RColorBrewer", "ggplot2", "Biotrings", as well as 421 shell scripts, in the analyses.

\section{Data availability statement}

423 The RNA-seq data of Crassostrea gigas were from GEO database (accession number 424 SRP014559) ${ }^{26}$, and the rest of species from DDBJ (accession number DRA003460) 
425 (Supplementary Data 1). All the codes used have been uploaded to GitHub: 426 https://github.com/guosongcn/evo-devo

\section{Acknowledgments}

428 We thank Michael Lachmann for inspiring this study, Zhisong He, Qian Li and 429 Qianhui Yu for helpful discussions, and Pavel Mazin for critical comments.

\section{$430 \quad$ Funding}

431 This work was supported by the National Natural Science Foundation of China 432 (Grants 31420103920 and 91331203 to P.K.); the National One Thousand Foreign

433 Experts Plan (Grant WQ20123100078 to P.K.); and Grant-in-Aid for Scientific 434 Research on Innovative Areas (17H06387 to N.I.).

\section{Author contribution}

436 S.G, HY.H and C.X designed and executed the bioinformatics analysis. S.G and PK 437 wrote the manuscript. N.I and P.K revised the manuscript. PK supervised the project.

\section{Conflict of interest}

439 The authors declare no conflict of interest. 


\section{References}

441 1. Von Baer KE. Uber Entwicklungsgeschichte Der Thiere. (1828).

443 2. Haeckel E. Generelle Morphologie der Organismen. Berlin: Georg Reimer, (1866).

3. Duboule D. Temporal colinearity and the phylotypic progression: a basis for the stability of a vertebrate Bauplan and the evolution of morphologies through heterochrony. Dev

4. Domazet-Loso T, Tautz D. A phylogenetically based transcriptome age index mirrors Suppl, 135-142 (1994).

5. Kalinka AT, et al. Gene expression divergence recapitulates the developmental hourglass

6. Irie N, Sehara-Fujisawa A. The vertebrate phylotypic stage and an early bilaterian-related stage in mouse embryogenesis defined by genomic information. BMC

7. Irie N, Kuratani S. Comparative transcriptome analysis reveals vertebrate phylotypic Biol 5, 1 (2007).

8. $\mathrm{Hu} \mathrm{H}$, et al. Constrained vertebrate evolution by pleiotropic genes. Nat Ecol Evol 1, $1722-1730$ (2017).

9. Quint M, Drost HG, Gabel A, Ullrich KK, Bonn M, Grosse I. A transcriptomic hourglass in plant embryogenesis. Nature 490, 98-101 (2012).

10. Cheng X, Hui JH, Lee YY, Wan Law PT, Kwan HS. A "developmental hourglass" in fungi. Mol Biol Evol 32, 1556-1566 (2015). 
11. Cridge AG, Dearden PK, Brownfield LR. Convergent occurrence of the developmental hourglass in plant and animal embryogenesis? Ann Bot 117, 833-843 (2016).

12. Bininda-Emonds OR, Jeffery JE, Richardson MK. Inverting the hourglass: quantitative evidence against the phylotypic stage in vertebrate development. Proc Biol Sci 270, 341-346 (2003).

13. Roux J, Robinson-Rechavi M. Developmental constraints on vertebrate genome evolution. PLoS Genet 4, e1000311 (2008).

14. Artieri CG, Haerty W, Singh RS. Ontogeny and phylogeny: molecular signatures of selection, constraint, and temporal pleiotropy in the development of Drosophila. $B M C$ Biol 7, 42 (2009).

15. Comte A, Roux J, Robinson-Rechavi M. Molecular signaling in zebrafish development and the vertebrate phylotypic period. Evol Dev 12, 144-156 (2010).

16. Piasecka B, Lichocki P, Moretti S, Bergmann S, Robinson-Rechavi M. The hourglass and the early conservation models--co-existing patterns of developmental constraints in vertebrates. PLoS Genet 9, e1003476 (2013).

17. Levin M, et al. The mid-developmental transition and the evolution of animal body plans. Nature 531, 637-641 (2016).

18. Uesaka M, Kuratani S, Takeda H, Irie N. Recapitulation-like developmental transitions of chromatin accessibility in vertebrates. Zoological Lett 5, 33 (2019).

19. Richardson MK, Keuck G. Haeckel's ABC of evolution and development. Biol Rev Camb Philos Soc 77, 495-528 (2002).

20. Jeffery JE, Bininda-Emonds OR, Coates MI, Richardson MK. Analyzing evolutionary patterns in amniote embryonic development. Evol Dev 4, 292-302 (2002). 
21. Li JJ, Huang H, Bickel PJ, Brenner SE. Comparison of D. melanogaster and C. elegans developmental stages, tissues, and cells by modENCODE RNA-seq data. Genome Res 24, 1086-1101 (2014).

22. Yanai I, Peshkin L, Jorgensen P, Kirschner MW. Mapping gene expression in two Xenopus species: evolutionary constraints and developmental flexibility. Dev Cell 20, 483-496 (2011).

23. Bozinovic G, Sit TL, Hinton DE, Oleksiak MF. Gene expression throughout a vertebrate's embryogenesis. BMC Genomics 12, 132 (2011).

24. Wagner RA, Tabibiazar R, Liao A, Quertermous T. Genome-wide expression dynamics during mouse embryonic development reveal similarities to Drosophila development. Dev Biol 288, 595-611 (2005).

25. Tomancak P, et al. Global analysis of patterns of gene expression during Drosophila embryogenesis. Genome Biol 8, R145 (2007).

26. Zhang G, et al. The oyster genome reveals stress adaptation and complexity of shell formation. Nature 490, 49-54 (2012).

27. Li B, et al. A Comprehensive Mouse Transcriptomic BodyMap across 17 Tissues by RNA-seq. Sci Rep 7, 4200 (2017).

28. Gao L, et al. Chromatin Accessibility Landscape in Human Early Embryos and Its Association with Evolution. Cell 173, 248-259 e215 (2018).

29. Sarropoulos I, Marin R, Cardoso-Moreira M, Kaessmann H. Developmental dynamics of lncRNAs across mammalian organs and species. Nature 571, 510-514 (2019).

30. Somel M, et al. Transcriptional neoteny in the human brain. Proc Natl Acad Sci U S A 106, 5743-5748 (2009). 
31. Litman T, Stein WD. Obtaining estimates for the ages of all the protein-coding genes and most of the ontology-identified noncoding genes of the human genome, assigned to 19 phylostrata. Semin Oncol 46, 3-9 (2019).

32. Yang Z, Nielsen R. Estimating synonymous and nonsynonymous substitution rates under realistic evolutionary models. Mol Biol Evol 17, 32-43 (2000).

33. Yang Z. PAML: a program package for phylogenetic analysis by maximum likelihood. Comput Appl Biosci 13, 555-556 (1997).

34. Sievers F, et al. Fast, scalable generation of high-quality protein multiple sequence alignments using Clustal Omega. Mol Syst Biol 7, 539 (2011).

35. Kanehisa M, Goto S. KEGG: kyoto encyclopedia of genes and genomes. Nucleic Acids Res 28, 27-30 (2000).

36. Alexa A, Rahnenfuhrer J. topGO: enrichment analysis for Gene Ontology. R Packag. version 2.26.0. $R$ Package version 2260, (2016).

37. Kumar S, Stecher G, Suleski M, Hedges SB. TimeTree: A Resource for Timelines, Timetrees, and Divergence Times. Mol Biol Evol 34, 1812-1819 (2017). 

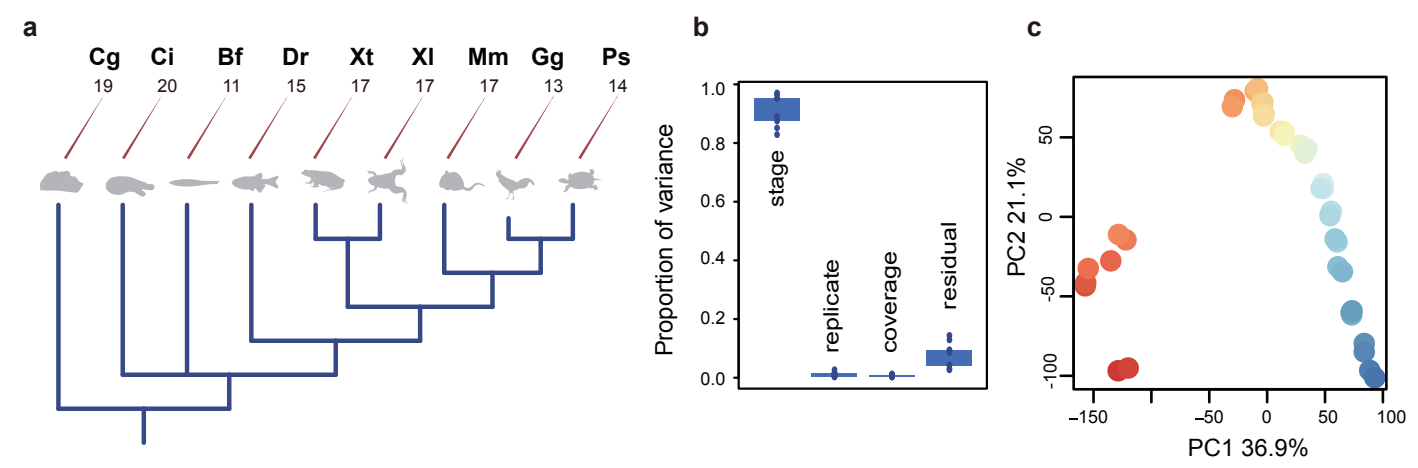

d
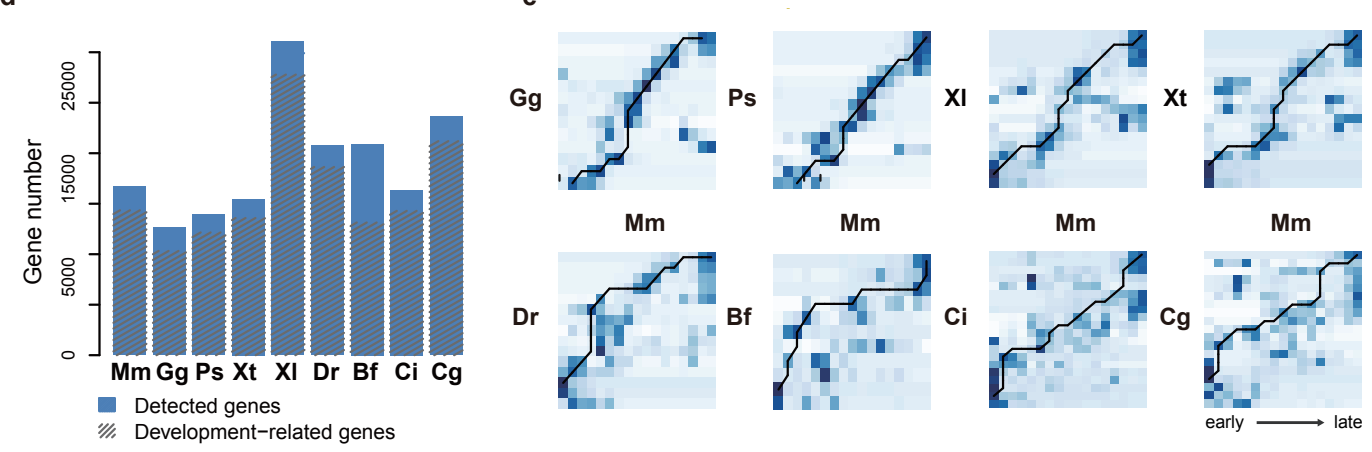

$\mathrm{Mm}$
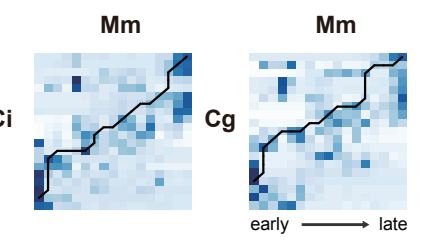

562 Figure 1. Species phylogeny and alignment of embryonic development stages.

563 a Experimental scheme showing the phylogenetic relationship among species (dark 564 blue dendrogram) and numbers of sampled developmental stages. The abbreviations 565 here and throughout the figures indicate species: $\mathrm{Cg}$ - oyster (Crassostrea gigas), Ci 566 - ciona (Ciona intestinalis), Bf - amphioxus (Branchiostoma floridae), Dr - zebrafish 567 (Danio rerio), Xt - Western clawed frog (Xenopus tropicalis), X1 - African clawed 568 frog (Xenopus laevis), Ps - turtle (Pelodiscus sinensis), Gg - chicken (Gallus gallus), $569 \mathrm{Mm}$ - mouse (Mus musculus). b Variance explained by different factors based on 570 ANOVA. Boxes represent the interquartile values of the variance proportion 571 explained by the factors listed on the $\mathrm{x}$-axis among nine species. Dots represent the 572 mean explained variance proportion for each species. c PCA plot based on the 573 expression of 16,685 genes in mouse development. Dots represent samples, and color 574 represents developmental stages (red - early, blue - late). d The number of detected 575 and development-related genes in each species. e Heatmap showing the pairing scores 576 reflecting overlap of stage-associate genes (darker shade of blue representing greater 577 overlap) and the optimal alignment path calculated using the Needleman-Wunsch 578 algorithm (black line) 


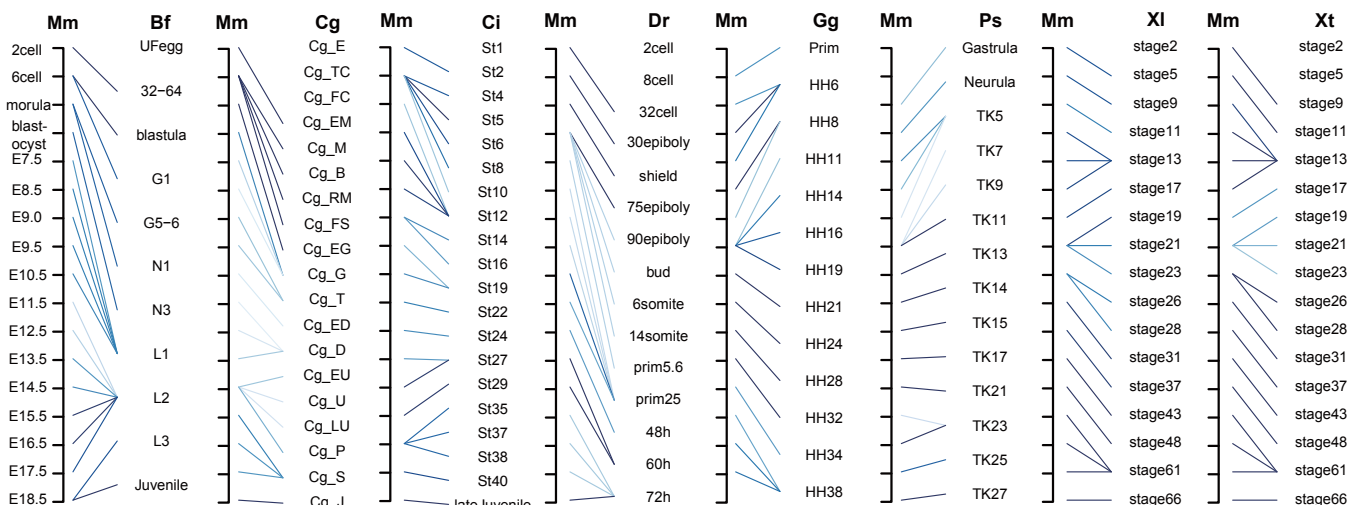

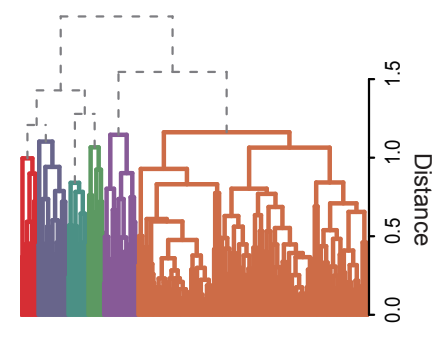

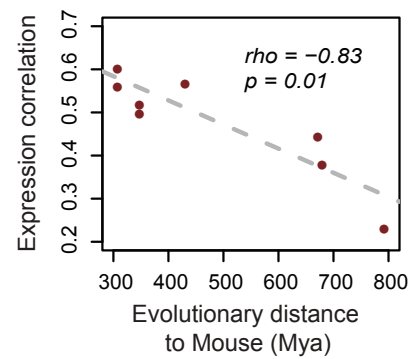

$\mathbf{f}$

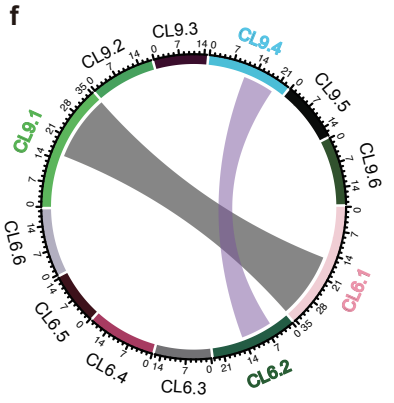

C
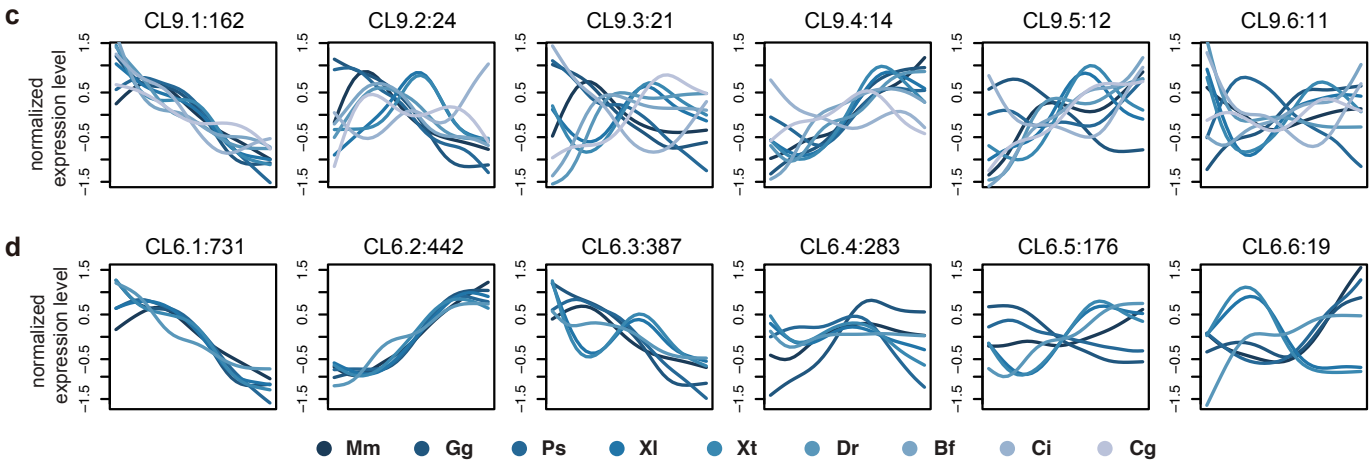

Figure 2. Developmental expression of genes based on the species' alignment.

581 a Pairwise alignment of developmental stages between mouse and the other species.

582 Thick lines represent alignments supported by technical replicates. Thicker lines

583 represent more stable alignments calculated by random subsampling of samples 500

584 times. b Hierarchical clustering of concatenated developmental gene expression

585 trajectories of 244 gene orthologs shared among nine species. Colors represent

586 clusters. c Developmental gene expression patterns in each of six clusters based on

587 nine-species gene orthologs. Colors represent species. Panel titles show cluster

588 identifiers and number of contained genes. d Developmental gene expression patterns in each of six clusters based on six vertebrate species gene orthologs. Panel titles 
590 show cluster identifiers and number of contained genes. e Relationship between the 591 similarity of developmental gene expression patterns and phylogenetic distances. The 592 expression similarity was calculated as the mean of Spearman correlation coefficients 593 between mouse and non-mouse expression trajectories. f Chord graph indicating the 594 relationship between clusters obtained using nine-species and six-species ortholog 595 genes. Wider chords represent stronger connection between clusters.

596 


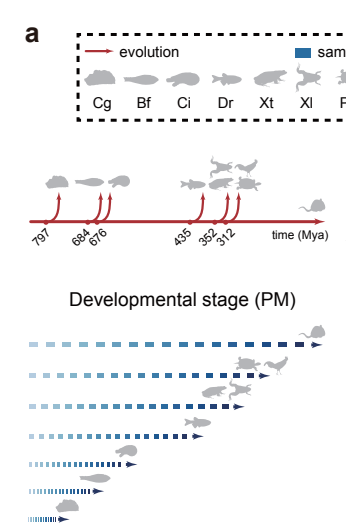

d

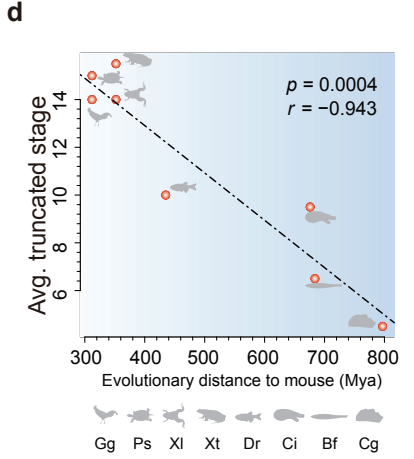

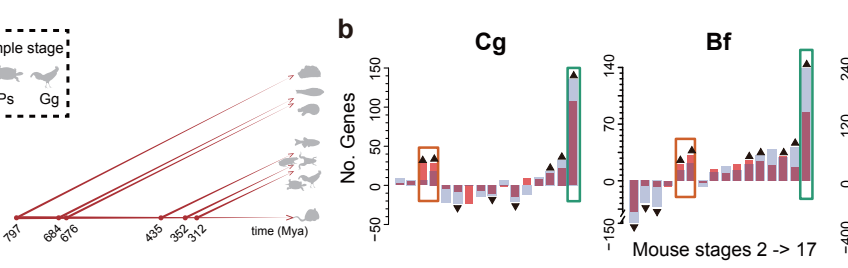
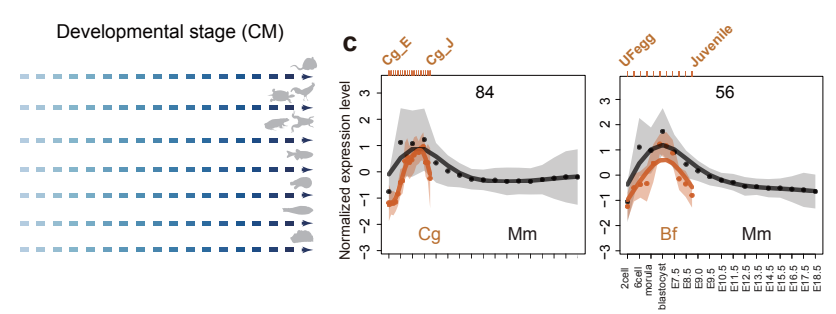

f

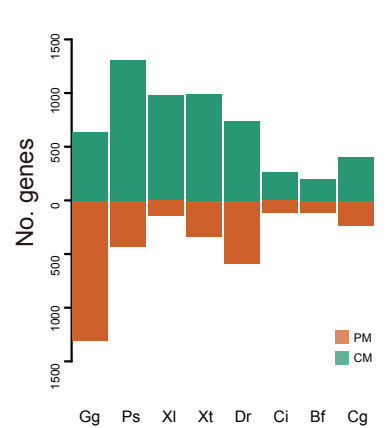

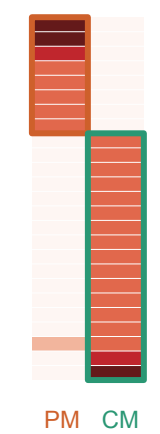
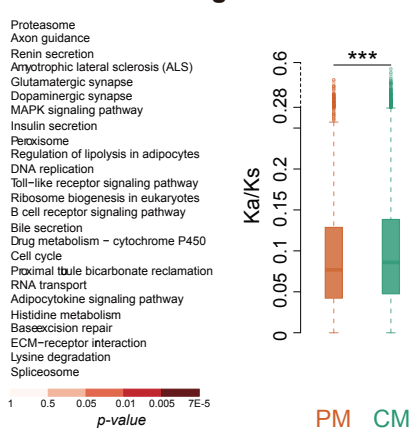

Figure 3. Relationship of developmental gene expression patterns among species.

599 a Schematic description of the progressive and continuous models and their predicted

600 alignment patterns. The progressive model (PM, left) involves an extension of 601 developmental program in more recently evolved species, thus predicting the best 602 alignment to a shortened mouse developmental course. The continuous model (CM, 603 right) predicts the best alignment to the complete developmental course. b Numbers

604 of genes showing the best expression trajectory's alignment between the complete 605 developmental course of the oyster (left), amphioxus (middle), and zebrafish (right) 606 and mouse developmental sets of different lengths: from two to 17 stages. Colors 607 indicate two methods used for developmental expression trajectory calculation: cubic 608 smooth spline (orange) and polynomial regression (gray). Colored rectangles mark 609 stages containing alignments fitting CM (green) or PM (orange) predictions. Black 610 triangles indicate the significantly greater (up) or lesser (down) number of genes than 611 that expected by chance aligning to an indicated mouse developmental interval 612 (permutation test, BH-corrected $p<0.05$ ). c Examples of PM gene expression 613 patterns. Dots show the cluster-level standardized expression levels at each 
614 developmental stage in mouse (black) and the other species (orange). The curves

615 represent average expression profiles, and the shaded regions represent the standard 616 deviation of curve estimates. d The relationship between the lengths of truncated sets 617 of mouse stages containing maximal numbers of PM genes for each of the eight 618 species and the phylogenetic distances to the mouse. Dots represent species. The 619 dotted line marks the regression curve. The Pearson correlation coefficient and linear 620 regression p-value are shown at the top right. Mya - millions of years ago. e Numbers 621 of CM and PM genes identified in each of the eight species. Note that PM gene 622 number in chicken $(\mathrm{Gg})$ might be inflated due to poor resolution of PM and CM 623 predictions at close phylogenetic distances. f KEGG pathways significantly enriched 624 in PM or CM from all eight non-mouse species. The heat map shows the p-values of 625 the enrichment tests. Colored rectangles mark significantly enriched KEGG pathways. 626 g Amino acid sequence conservation $(\mathrm{Ka} / \mathrm{Ks})$ of $\mathrm{PM}$ and $\mathrm{CM}$ genes from all eight 627 non-mouse species. Asterisks indicate the significance of the difference (Wilcoxon 628 rank-sum one-sided test, $p<0.0005$ ). 


\section{Supplementary Files}

This is a list of supplementary files associated with this preprint. Click to download.

- SupplementaryFigurecb.pdf

- SupplementaryData1.xlsx

- SupplementaryData2.xlsx

- SupplementaryData3.xlsx

- SupplementaryData4.xlsx

- SupplementaryData5.xlsx

- SupplementaryData6.xlsx

- SupplementaryData7.xlsx

- SupplementaryData8.xlsx

- SupplementaryData9.xlsx

- SupplementaryData10.xlsx 